\title{
Classification and recognition of handwritten digits by using mathematical morphology
}

\author{
V VIJAYA KUMAR ${ }^{1}$, A SRIKRISHNA ${ }^{2}$, B RAVEENDRA BABU ${ }^{2}$ \\ and M RADHIKA MANI ${ }^{3}$
}

${ }^{1}$ Department of Computer Science and Engineering and Information Technology, Godavari Institute of Engineering and Technology, Rajahmundary 533296

${ }^{2}$ Department of Computer Science and Engineering and Information Technology, Rayapati Venkata Ranga Rao (RVR) and Jagarlamudi Chandramouli (JC) College of Engineering, Guntur 522109

${ }^{3}$ Department of Computer Science and Engineering, Godavari Institute of Engineering and Technology, Rajahmundary 533296

e-mail: vakulabharanam@hotmail.com; atlurisrikrishna@yahoo.com;

rbhogapathi@yahoo.com; radhika_madireddy@yahoo.com

MS received 4 July 2009; revised 13 March 2010; accepted 2 June 2010

\begin{abstract}
The present paper proposes a novel algorithm for recognition of handwritten digits. For this, the present paper classified the digits into two groups: one group consists of blobs with/without stems and the other digits with stems only. The blobs are identified based on a new concept called morphological region filling methods. This eliminates the problem of finding the size of blobs and their structuring elements. The digits with blobs and stems are identified by a new concept called 'connected component'. This method completely eliminates the complex process of recognition of horizontal or vertical lines and the property called 'concavities'. The digits with only stems are recognized, by extending stems into blobs by using connected component approach of morphology. The present method has been applied and tested with various handwritten digits from modified NIST (National Institute of Standards and Technology) handwritten digit database (MNIST), and the success rate has been given. The present method is also compared with various existing methods.
\end{abstract}

Keywords. Region filling; connected components; blob(s); stem(s); thinning.

\section{Introduction}

Automatic handwriting recognition has a variety of applications at the interface between man and machine. The performance of a method for handwriting recognition can be evaluated by several of criteria, including size of the digit, independence of the writing style, reliability and speed of recognition. Recognition of handwritten digits is difficult because of the high variability of the scanned image. This is caused by the peculiar writing style of different 
persons, the context of the digit, different writing devices and media. This leads to scanned digits of different size and slant, and strokes that vary in width and shape.

The problem of handwriting recognition has been studied for decades and many methods have been developed. According to Liu et al (2003) handwritten digit recognition can be classified into two categories: offline recognition and online recognition. Offline recognition mainly processes and recognizes the user input handwritten digit, based on images (the scanned images of handwritten digit or the digital images transformed from the real handwritten). Many methods have been proposed to solve offline recognition by Khorsheed (2002). Inversely, the on-line recognition technology proposed by (Vuori et al 1999; Artieres et al 2000), which emerged in recent years, uses the geometry and temporal dynamics information of the users' input.

Mathematical morphology is an important tool that can be used to process images based on the shape information. Not much literature on morphology is existing on methods that exploit the technology for digit recognition. Haralick \& Kanungo (1990) presented their partially completed work of character recognition using mathematical morphology. The basic operations of erosion and dilation are discussed in Haralick \& Kanungo (1990) and their applications to recognize six out of ten handwritten digits. Albiol et al (2004) described the application of mathematical morphology to the problem of extracting the characters from a license plate in order to read it automatically. In their paper, new morphological operators are defined and described. Kim et al (1999) used mathematical morphology to decompose Chinese characters into their constituent strokes. Zhu et al (2000) proposed a character processing system that handles characters for which fonts are not provided on the terminals i.e. undefined characters. In the above mentioned paper they used morphology, to analyse the structures of the targeted undefined characters, to generate codes which describe the shapes of the characters based on the analysis results, and to reconstruct the characters from these codes. Badr \& Haralick (1995) described in their paper the design and implementation of a system that recognizes machine-printed Arabic words without prior segmentation. The technique is based on describing symbols in terms of shape primitives. At the time of recognition the primitives are detected on a word image using mathematical morphology operations. The system then matches the detected primitives with symbol models. Gu Lixu et al (1996) extracted characters from scene images using mathematical morphology. Barrera Junior et al (1998) presented the prototype of optical character reader (OCR). The characteristic of this system is that all the necessary image processing tasks are performed by mathematical morphology operators. Eugene and Edward (1994) developed a class of structuring-element pairs for segmentation-free character recognition via the morphological hit-or-miss transform for Courier font. Both hit and miss structuring elements are selected so that the hit-or-miss transform can be applied across the test image without prior segmentation. Badr \& Haralick (1994) described a new method for recognizing cursive and degraded text using OCR technology. Using this method, symbols on a page are identified by detecting primitives (parts of symbols), using mathematical morphology operations, in a way that does not require or involve a prior segmentation step. Lixu et al (1998) described that since characters are composed from thin and long lines, the character regions in an image can be easily detected and extracted according to their thin and long features. In their paper, they proposed the new approach of detecting regions with different widths (widths of character lines) so as to extract characters from cover images using mathematical morphology. Mohammad \& Husain (2007) described a method to recognize handwritten digits by extending the work done by Haralick \& Kanungo (1990). This paper attempts to enhance it to apply it for the remaining four digits. 
A blob is represented as a drop or splotch and occurs when holes are filled. Blobs are found by filling any possible hole that may occur in the image. To count the number of blobs in the image, the original image is subtracted from the filled image. Number of objects present in the difference is an indication of the number of blobs in the objects. Other than blob, in the original image is called the stem.

The above authors recognized the blobs by selecting a structuring element that is big enough to connect the strokes. This method of selecting structuring element is a tedious process. The present paper completely eliminates this process. The above authors followed a tedious process in recognizing stems by identifying horizontal and vertical lines with different orientations.

The proposed method recognizes all the ten digits by using the morphological decision tree with the help of morphological operator's dilation, thinning, region filling and connected component approach to extract various features and check for various topological configurations. The paper is organized as follows. The proposed algorithm is described in section 2 . Section 3 contains experimental results and discussions and conclusions are given in section 4 .

\section{Methodology}

There are ten digits in English language and each digit is differentiated from the other digits by some characteristic feature(s). Recognition of the ten numerals appears simple at first. However, the problems that arise due to similarities between different numerals and discrepancies between the same numeral must be tackled by analysing the similar and dissimilar features and then decisions should be made accordingly. The present paper divided the ten digits of English language into two groups. Group 1 consists of digits with blobs with/with out stems. This group consists of digits $\{0,4,6,8$, and 9$\}$. Group 2 consists of digits with only stems, digits $\{1,2,3,4,5$, and 7$\}$. The group 1 is further divided in to two subgroups i.e. the digits with only two blobs $\{8\}$ and another with a single blob with or without stems $\{0,4,6$ and 9$\}$. The blobs are identified by region filling method which is different from previous methods. For this purpose, blobs are initially filled by using morphological region filling method as described below.

Let A, denote a set containing a subset whose elements are 8-connected boundary points of a region. Beginning with a point $p$ inside the boundary, the objective was to fill the entire region with 1's. Assign a value 1 to $p$ to begin. The region is filled with 1's using the equation (1).

$$
X_{k}=\left(X_{k-1} \oplus B\right) \cap A^{c}
$$

$k=1,2,3 \ldots$, where $X_{o}=p$, and $B$ is the $3 \times 3$ symmetric structuring element. The algorithm terminates at iterative step $k$ if $X_{k}=X_{k-1}$. The set union of $X_{k}$ and $A$ contains the filled set and its boundary.

Once the holes are filled, the original image is subtracted from the filled image and the number of objects present is determined by a connected component approach which is different from previous methods as described by equation (2).

Let $Y$ represent a connected component contained in a set $A$ and assumes that a point $p$ of $Y$ is known. Then the following iterative expression yields all the elements of $Y$ :

$$
X_{k}=\left(X_{k-1} \oplus B\right) \cap A
$$

$k=1,2,3 \ldots$, where $X_{o}=p$, and $B$ is the $3 \times 3$ structuring element with all 1's. If $X_{k}=$ $X_{k-1}$, the algorithm has converged and let $Y=X_{k}$. 


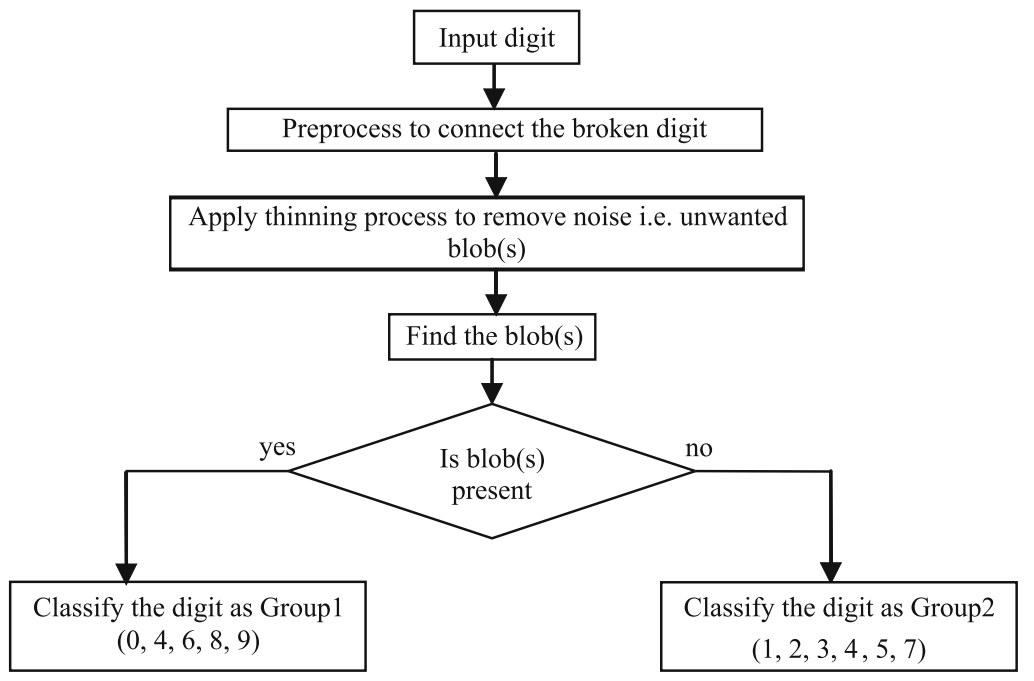

Figure 1. Decision tree for English numerals.

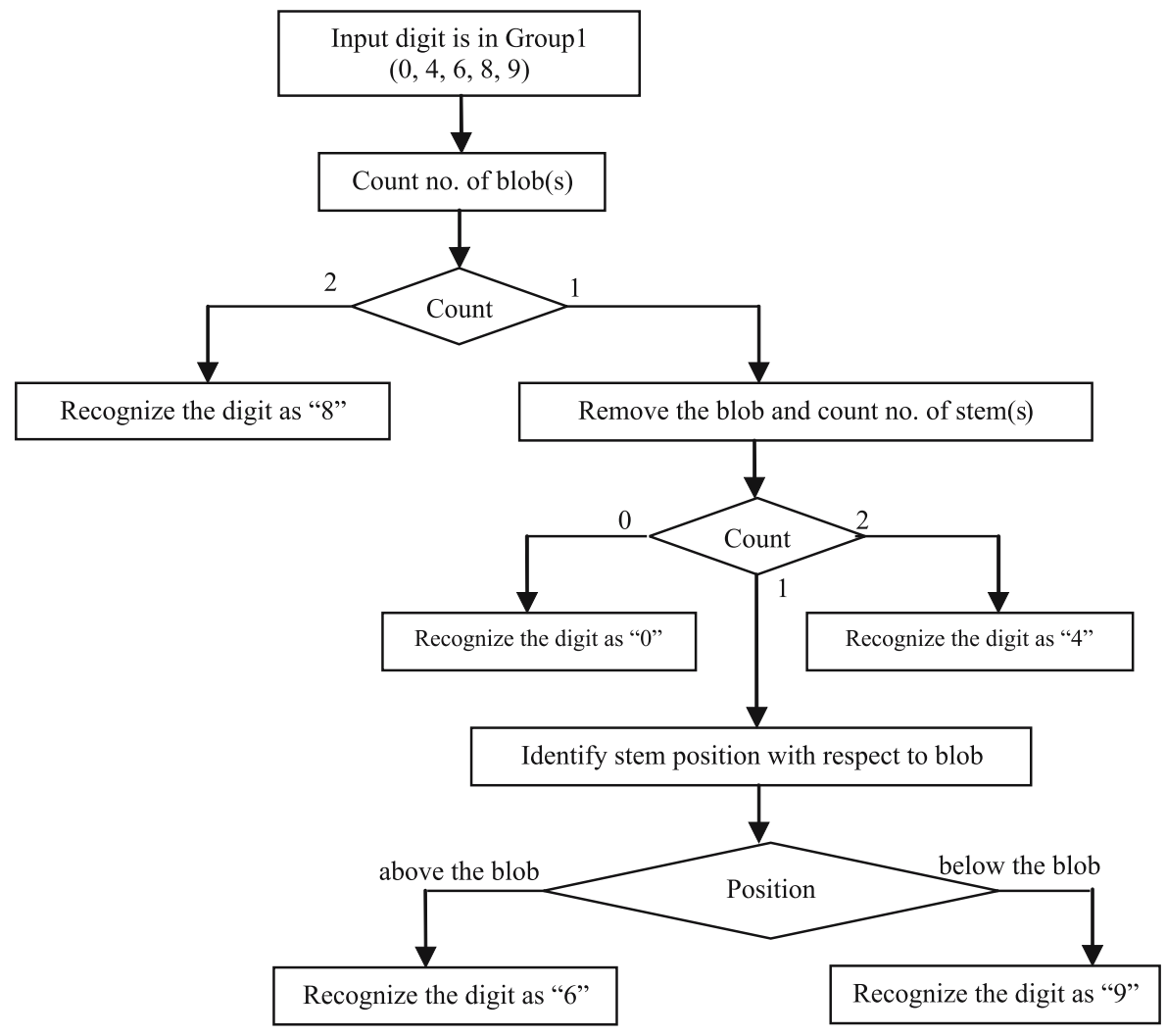

Figure 2. Decision tree for group 1 numerals. 


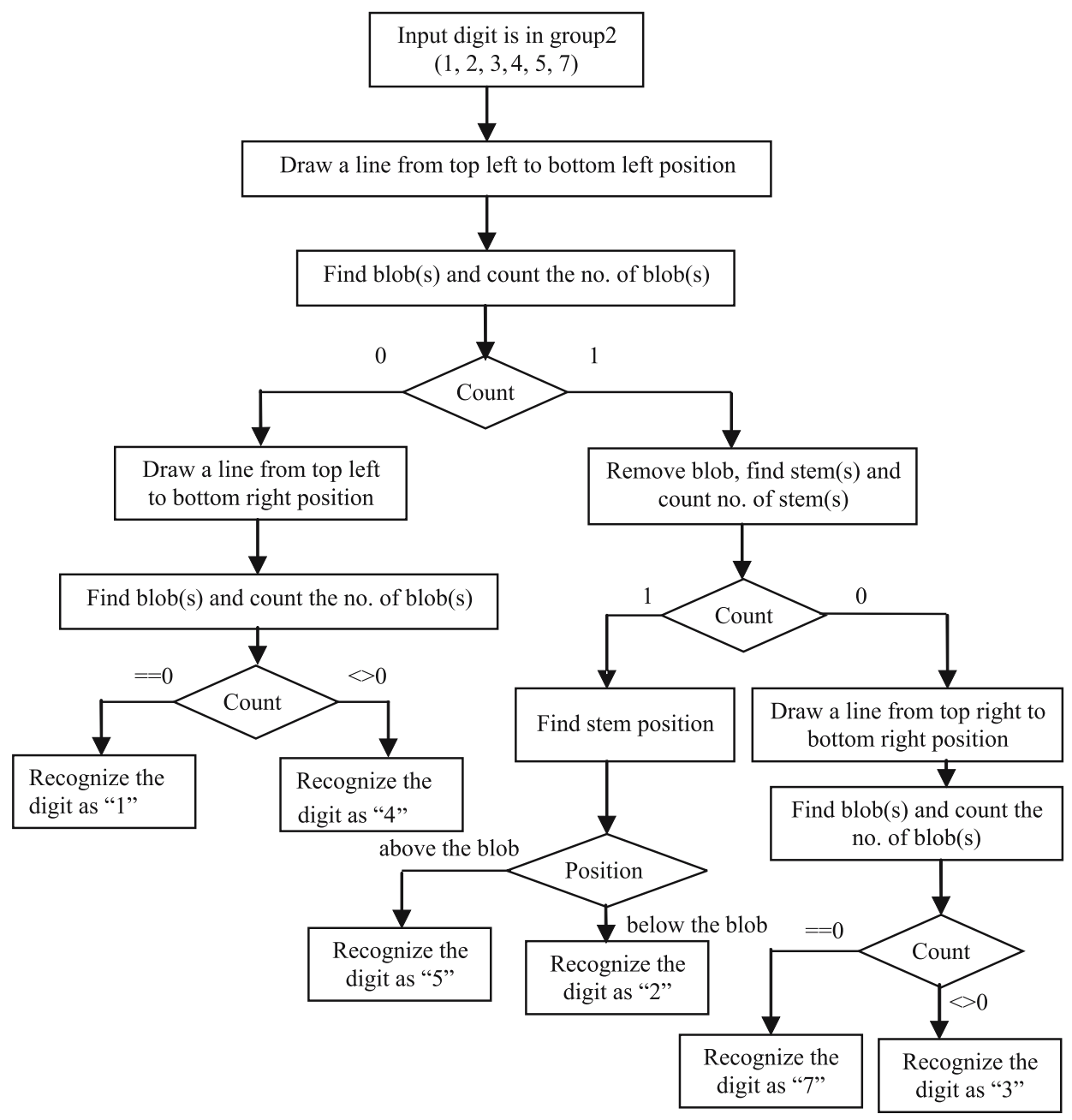

Figure 3. Decision tree for group 2 numerals.

By equation (2) a connected component blob is identified and later it is filled by a Region filling algorithm with background intensity. To check if there is any further blob a scan line approach is used to identify any fore ground pixel is in the image zone. Then again the above process of equation (2) is repeated to identify whether it is a connected component or not. The process terminates when all the connected components are identified.

The process of recognizing these digits are given in detail in the flowchart in figures 1,2 and 3. To overcome the disadvantage caused due to breaks in the handwritten digits initially, dilation technique is applied. To overcome the noise created by extra blobs in the form of extra single or more dots, thinning or skeletonization is applied as a basic step.

\section{Experimental results and discussions}

Recognition of digits is aided by the use of morphological decision tree. In group 1 blobs are identified by the above said process and numbers of blobs are counted. Based on the number 


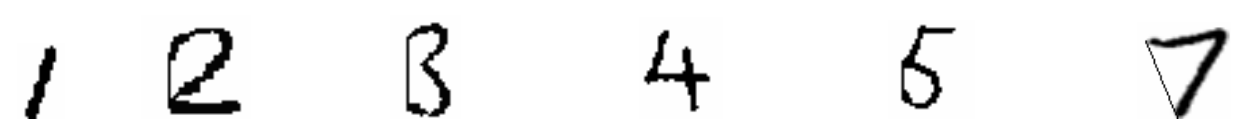

Figure 4. Line drawn from top left pixel position to the bottom left position of the digit.
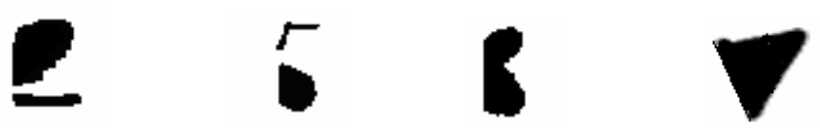

Figure 5. Blobs and stems of subgroup 2-b.

of blobs, the group 1 digits are categorized in to two subgroups. A digit with two blobs is identified as $\{8\}$ and the digits with one blob i.e. $\{0,4,6$, and 9$\}$ are categorized as subgroup $1 b$. They are further recognized by looking at number of stems and their position. The digits with blobs and stems are identified by removing blob portion from the digit. The digit with zero stems is identified as $\{0\}$ and with two stems is identified as $\{4\}$ and digits with one stem i.e. $\{6,9\}$ are identified based on the position of the stem with respect to the blob that is either above the blob or below the blob. For $\{6\}$ the stem appears above the blob and for $\{9\}$ stem appears below the blob. The digit four can be written as $\{4$ or 4$\}$. By this the first digit four can be placed in group 1 and the second four will be placed in group 2 .

The group 2 digits that consist of only stems are recognized by the following novel method. Initially, a line is drawn from top left pixel position of the digit to the bottom left position of the digit. By this blobs will be formed for digits $\{2,3,5$, and 7$\}$ as shown in figure 4 .

In group 2 , blobs are identified by the above said process. Then, from the original image blob is removed and based on the position of the stem the subgroup $2 b$ is identified as $\{2$ or 5$\}$ as shown in figure 5. For $\{2\}$ the stem appears below the blob and for $\{5\}$ the stem appears above the blob. The digits $\{3$ and 7$\}$ are identified as subgroup $2 \mathrm{c}$ which gave no stems and with single blob as shown in figure 5. To identify individual digits in this subgroup a line will be drawn from top left pixel position to the bottom right pixel position as shown in figure 6. If no blobs are identified then it is treated as $\{1\}$ and if blobs are identified then it is recognized as $\{4\}$. To identify $\{3$ and 7$\}$, a line is drawn from top right pixel position to bottom right pixel position as shown in figure 7 . If a blob is identified then digit $\{3\}$ is recognized. In this case for digit $\{7\}$ no blob will be formed. The digit $\{1$ and 4$\}$ is treated as subgroup 2a where no blobs will be formed by connecting the top left pixel position to the bottom left position of the digit as shown in figure 4 . The performance of the algorithm is compared with the algorithm of (Haralick \& Kanungo 1990; Mohammad \& Husain 2007). In Haralick \& Kanungo (1990) the authors recognized only six digits $\{2,3,4,5$, 6, and 9\} by assuming that if there is a single blob it can be either $\{6\}$ or $\{9\}$. However, they have not considered the case of the digit $\{0\}$, which has a single blob. In Mohammad \& Husain (2007)

\footnotetext{
Figure 6. Line drawn from top left pixel position to the bottom right position of the digit.
}

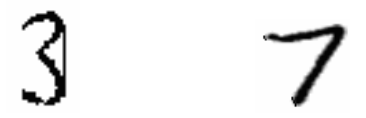

Figure 7. Line drawn from top right pixel position to the bottom right position of the digit. 
Table 1. The recognition rate of handwritten English numerals for 20 user(s).

\begin{tabular}{lc}
\hline Digits & $\%$ of success rate \\
\hline "0" & $95 \%$ \\
"1" & $95 \%$ \\
"2" & $90 \%$ \\
"3" & $95 \%$ \\
"4" & $90 \%$ \\
"5" & $95 \%$ \\
"6" & $90 \%$ \\
"8" & $85 \%$ \\
"9" & $100 \%$ \\
\hline
\end{tabular}

the authors recognized the remaining digits by predicting initially whether there is a single blob or two blobs. If there are no blobs, handwritten digit was tested for $\{1\}$ and $\{7\}$. If there is a single blob it has recognized it as $\{0\}$, but not considered the cases $\{6\}$ and $\{9\}$.

Here, we present results on the MNIST (Modified National Institute of Standards and Technology) handwritten digit database, which consists of 60,000 training set and 10,000 test digits. In the experiments, we have used 20 handwritten digit sets of MNIST database. For each digit set, five training digits and five test digits of MNIST database are taken. This gives a total of 200 test digits for our experiments. The recognition of success rate of each digit is shown in the table 1 .

\section{Conclusions}

The present algorithm is superior to previous algorithms because it has evolved a new technique of recognition of digits by identification of blobs and stems. Most of the digits are recognized by identification of blob(s) and to possible extent stems are extended to blob(s) by specific rules as described in section 2 . The average success rates of recognition of all digits are above $90 \%$. The present method fails in detecting the broken digits with large gap, incomplete digits and the digits with extra stems or strokes. This is recommended for the future work.

The authors would like to express their gratitude to Sri K V V Satya Narayana Raju, Chairman, and K Sashi Kiran Varma, Managing Director, Chaitanya group of Institutions for providing necessary research infrastructure. They would like to thank Dr G V S Ananta Lakshmi for her invaluable suggestions and constant encouragement which led to improvise the presentation quality of this paper.

\section{References}

Albiol Antonio, Mossi J M, Albiol Alberto, Naranjo V 2004 Automatic license plate reading using mathematical morphology. Proceeding (452) Visualization, Imaging, and Image Processing Spain 
Artieres T, Marchand J M, Gallinari P et al 2000 Stroke level modelling of on-line handwriting through multi-modal segmental models. IWFHR

Badr Al-Badr, Haralick, Robert M 1994 Symbol recognition with out prior segmentation. SPIE 2181: 303-314

Badr B Al, Haralick R M 1995 Segmentation free word recognition with application to Arabic. Proceedings of the Third International Conference on Document Analysis and Recognition (Volume 1) IEEE Computer Society

Barrera Junior, Terada Routo Lotufo Roberto De A, Hirata Nina, Hirata Roberto, Zampirolli F A 1998 OCR based on mathematical morphology. J. Proc. SPIE Non-Linear Image Processing IX 197-208

Eugene J Kraus, Edward R Dougherty 1994 Segmentation-free morphological character Recognition. SPIE 2181: 14-23

Gu Lixu, Tanaka Naoki, Kaneko Toyohisa 1996 The extraction of characters from scene image using mathematical morphology IAPR Workshop on Machine Vision Applications Tokyo, Japan November 12-14

Haralick Robert M, Kanungo Tapas 1990 Character recognition using mathematical morphology. Proceedings of the United States Postal Service Advanced Technology Conference, November 5-7, 2: 973-986

Khorsheed M S 2002 Off-line Arabic character recognition. A Review, Pattern Analysis and Application 5: 31-45

Kim Jin Wook, Kim Kwang In, Choi Bong Joon, Kim Hang Joon 1999 Decomposition of Chinese characters in to strokes using mathematical morphology. (Oxford UK: Elsevier Science Ltd.) NonLinear Analysis 20(3):

Liu Cheng-Lin, Nakashima Kazuki, Sako Hiroshi et al 2003 Handwritten digit recognition: Benchmarking of state-of-the-art techniques. Pattern Recognition 36: 2271-2285

Lixu Gu, Naoki Tanaka, Toyohisa Kaneko, Haralick R M 1998 The extraction of characters from cover Images using mathematical morphology. Systems and Computers in Japan 29(24): 33-42

Mohammad Fatimah, Husain S A 2007 Character recognition using mathematical morphology. Proceedings of the International Conference on Electrical Engineering-ICEE

Vuori Voukko, Laaksonen Jorma, Oja Erkki et al 1999 On-line adaptation in recognition of handwritten alphanumeric characters. Fifth International Conference on Document Analysis and Recognition Bangalore India 792

Zhu Oing, Kodate Akihisa, Kim Woontae, Urano Yoshiyori, Tominaga Hideyoshi 2000 An undefined character processing system using structure analysis by mathematical morphology. Systems and Computers in Japan 31(9): 60-71 